\title{
Elaboration and knowledge acquisition
}

\author{
BARRY S. STEIN \\ Tennessee Technological University, Cookeville, Tennessee \\ JOAN LITTLEFIELD and JOHN D. BRANSFORD \\ Vanderbilt University, Nashville, Tennessee \\ and \\ MARTIN PERSAMPIERI \\ Florida State University, Tallahassee
}

\begin{abstract}
Three experiments that explored the effects of different types of elaboration on knowledge acquisition are presented. The materials used were adapted from Stein and Bransford (1979) and were designed to simulate conceptual relationships encountered by people working in an unfamiliar domain. These materials were supplemented with elaborations that were designed to increase the distinctiveness of the memory trace, increase the distinctiveness of the memory trace while maintaining a high level of associative relatedness to key concepts, or reduce the arbitrariness of relationships among key concepts. The results replicate and extend previous research by showing that elaborations that reduce the arbitrariness of relationships facilitate both cued recall and recognition performance but that other types of elaborations appear to have little effect on performance. The implications of these results for theories of elaboration and knowledge acquisition are discussed.
\end{abstract}

One problem that is often faced by a novice who is trying to understand a new domain is that there are many new relationships involving concepts, facts, or rules that seem arbitrary and confusing. For example, a biology novice trying to learn about veins and arteries may find it difficult at first to understand and remember that arteries have thick walls, are elastic, and do not have valves, whereas veins are less elastic, have thinner walls, and have valves (see Stein \& Bransford, 1979). It is easy to confuse these relationships, just as a person who knows little about baseball might have trouble remembering the difference between a force-out and a sacrifice play (e.g., Chiesi, Spilich, \& Voss, 1979). Considering the crucial role that domain-related knowledge can play (see Auble, 1982, for a review), it is important to understand how people acquire expertise and use it effectively.

One area of research that seems applicable to these concerns involves the concept of elaboration (e.g., Anderson \& Reder, 1979; Craik \& Tulving, 1975; Rohwer, 1966, 1980). Elaboration research has emphasized the role that prior experience and knowledge can play in acquiring new information. In particular, recent studies that delineate the conditions under which prior knowledge may facilitate learning seem relevant to understanding how people may

This research was supported in part by N.I.E. Grant G-79-0117. We would like to thank Ronald Fisher and John Black for their helpful comments on an earlier version of this manuscript. Some of this research was presented at the meeting of the Southeastern Psychological Association in New Orleans, 1984. Requests for reprints should be sent to Barry S. Stein, Psychology, Box 5047, Tennessee Technological University, Cookeville, TN 38505. use available knowledge to facilitate retention and to acquire additional expertise.

The results of several recent studies support the hypothesis that retention is facilitated by acquisition conditions that prompt people to elaborate information in a way that increases the distinctiveness of their memory representations. Several investigators have manipulated distinctiveness by varying the number of different decisions that subjects are asked to make about target concepts (e.g. Johnson-Laird \& Bethell-Fox, 1978; Ross, 1981). Other investigators have manipulated distinctiveness by presenting sentence contexts that prompt subjects to consider varying numbers of properties and events that are related to target concepts. For example, Craik and Tulving (1975) embedded target concepts (e.g., WATCH) in sentence contexts that varied in complexity. A relatively simple context might involve "I lost my WATCH," whereas a more complex context might involve "The old man hobbled across the room and picked up the valuable WATCH from the mahogany table." Craik and Tulving found higher free recall for target concepts that had been embedded in more complex sentence contexts. Differences between simple and more complex acquisition contexts were even greater when subjects were asked to remember the target concepts after being supplied with the sentence contexts in which the concepts had originally occurred.

Not all attempts to improve retention by supplying elaborate information have met with success. For example, Chiesi et al. (Experiment 5, 1979) and Stein and Bransford (1979) have found that supplying elaborations 
that may increase trace distinctiveness sometimes debilitates retention. Fisher and Craik (1980) have also noted conditions in which distinctive elaborations do not facilitate recognition test performance. They have subsequently argued that in order for distinctive elaborations to facilitate retention, people must be able to redintegrate (reinstate) the encoding context at the time of test. These investigations imply that two distinct factors mediate the effects of elaboration activities on retention. One factor is the distinctive information content of the elaboration, and the second factor involves the learner's ability to reinstate the encoding context by using cues provided at the time of test. Fisher (1981) and Fisher and Craik (1980) have manipulated redintegration of the original encoding context by varying the amount of distinctive elaborative information that is provided at the time of retrieval. They found that distinctive elaborations facilitated the retention of target concepts when sufficient cues were provided during retrieval to reinstate the encoding context.

Other research findings suggest that redintegration does not necessarily occur even when distinctive acquisition information is provided at the time of test. For example, previous investigations by Stein, Morris, and Bransford (1978) found that distinctive elaborations did not necessarily facilitate retention even when the entire encoding context (minus the target concept) was presented as a cue for recall. One possible explanation for these results is that the distinctive elaborations did not facilitate retention because of a lack of integration between elaborative information and target concepts during encoding. It therefore appears necessary to examine additional variables that might clarify the conditions under which elaborations and target concepts are connected.

One method for increasing the probability of integration and redintegration is to use elaborative information that is associatively related to the target concepts (Fisher $\&$ Craik, 1980). For example, to assure integration of elaborations and target concepts, Fisher and Craik used highly associated words and concepts (e.g., "He washed in the bath"). In contrast, a low-integration sentence might be "He took a bath.") An alternative to using associated relatedness as a means of increasing integration was proposed by Stein and Bransford (1979). They argued that elaborations will facilitate retention when the information provided makes relationships between target concepts and the retrieval cues less arbitrary. For example, given a statement such as "The fat man read the sign" and a retrieval cue such as "Which man read the sign?," elaborations such as "The fat man read the sign warning about thin ice" should improve retention because the information on the sign is especially relevant to an overweight person. Elaborations such as "The fat man read the sign warning to keep off the grass" are clearly much less effective in reducing the arbitrariness of the relationship between "fat" and "sign reading"; consequently, they would not be expected to improve retention even though they increase the distinctiveness of the sentence. Stein and Bransford argued that elaborations that clarify the significance of relationships in sentences help integrate the information and improve retention when appropriate cues are provided. Although this theoretical position does not implicate associative relatedness as a prerequisite for integration, the materials used by Stein and Bransford (1979) often included concepts that are associatively related to target concepts (e.g., fat-thin) in the nonarbitrary elaboration conditions. Therefore, it is difficult to determine whether associative relatedness is a necessary and perhaps sufficient condition for explaining the facilitative effects of nonarbitrary elaborations.

The present study sought to extend previous investigations by Stein et al. (1978) and Stein and Bransford (1979) to evaluate the effects that associative relatedness has on arbitrary and nonarbitrary elaborations. To mimic the problem of apparent arbitrariness that is often faced by novices learning about a new domain, the present study included lists of base sentences similar to those used in previous research by Stein and Bransford (1979): “The bald man used the telephone"; "The funny man bought the ring",; "The hungry man bought the tie"; "The short man picked up the broom"; " The strong man read the book." These base sentences are constructed so that the relationship between each type of man and the action performed seems arbitrary; there is no particular reason for a certain type of man to perform a particular action. The present experiments explored the effects of supplying different types of elaborations for these sentences in order to obtain a better understanding of the knowledge that people must activate in order to understand and remember potentially confusing information. Specifically, the experiments that follow examined the degree to which retention is primarily affected by (1) elaborations that increase distinctiveness by enhancing trace complexity, (2) elaborations that increase trace complexity by adding information that is associatively related to target concepts, and (3) elaborations that increase what Stein and Bransford (1979) called " precision," that is, the degree to which elaborations clarify the significance of target concepts.

\section{EXPERIMENT 1}

In Experiment 1, learners were presented with several different types of elaborations that increased the distinctiveness of the encodings for target concepts by supplying additional (nonredundant) semantically congruous information. These elaborations were added to a set of base sentences that were constructed to simulate arbitrary and confusing relationships (e.g., "The short man picked up the broom," "The tall man purchased the crackers," "The fat man read the sign," etc.).

Two variables, the relatedness of elaborations to target concepts and the precision of elaborations, were orthogonally varied to produce four different types of elaborations. The relatedness of elaborations to target concepts was manipulated by adding elaborative phrases to base sentences that contained concepts or phrases highly 
related to the target concepts or by adding elaborative phrases that contained concepts or phrases judged to be less related to the target concepts. The precision of elaborations was manipulated by supplying elaborative phrases that clarified or did not clarify the significance of the target concepts (e.g., short) in the sentence concept (e.g., "The short man picked up the broom to operate the light switch" or "The short man picked up the broom to sweep the floor'). It was hypothesized that only precise elaborations would facilitate retention irrespective of whether the target-elaboration associative relatedness was relatively high or relatively low.

\section{Method}

Subjects. Eighty-four undergraduates enrolled in introductory psychology courses at Tennessee Technological University participated for course credit.

Materials. Two sets of sentence types were constructed. Each set contained 10 base sentences (base), 10 precise elaborations of these base sentences (PE), and 10 imprecise elaborations of these base sentences (IE). In the first set, PE and IE sentences were constructed so that there would be a relatively high degree of associative relatedness between target concepts and words in the elaborative phrases. We refer to this as the high-related (HR) set. In the second set, PE and IE sentences were constructed so that there would be a lower degree of associative relatedness among target concepts, base-sentence contexts, and words in the elaborative phrases. We refer to this as the low-related (LR) set. Examples of base, PE, and IE sentences for the HR set are: "The funny man bought the ring," "The funny man bought the ring that squirted water," and "The funny man bought the ring and told a joke." Examples of base, PE, and IE sentences for the LR set are: "The short man picked up the broom," "The short man picked up the broom to operate the light switch," and "The short man picked up the broom to sweep the floor." Both the HR and LR sentence sets are presented in Appendix 1 .

Twenty-five subjects were given the target words (adjectives describing the type of man) together with key concepts or phrases used in the PE and IE concepts for both the HR and LR sets and were asked to rate the relatedness of each pairing on a scale of $1-5$ (with 5 corresponding to high relatedness). The mean ratings for PE and IE contexts in the HR set were 3.20 and 3.71 , respectively. The mean ratings for the $P E$ and IE contexts in the LR set were 1.56 and 1.49 , respectively. A $2 \times 2$ analysis of variance on the rating scores revealed a significant effect for relatedness (LR vs. HR) $\left[\mathrm{F}(1,72)=4.67, \mathrm{p}<.001, \mathrm{MS}_{\mathrm{Res}}=.200\right]$, indicating that $\mathrm{HR}$ sentences were generally rated higher than LR sentences. There were also significant effects for the type of elaboration (PE vs. IE) and the interaction (PE-IE $\times$ HR-LR) $[\mathrm{F}(1,72)=6.15$, $\mathrm{p}<.05$, and $F(1,72)=10.5, \mathrm{p}<.01$, respectively]. These latter findings are a result of the fact that IE sentences in the HR set were rated significantly higher than PE sentences in the HR set $[F(1,72)=$ $16.4, p<.01$ ] There were no significant differences between the ratings for IE sentences and PE sentences in the LR set $[F(1,72)=.31$, $\mathrm{p}>$.10].

Procedure. The subjects were given incidental learning instructions. They were told that the purpose of the experiment was to obtain normative data about sentence comprehensibility. All subjects were instructed to rate the comprehensibility of each sentence on a scale of 1 to 5 , with 1 being the lowest rating.

The treatment for subjects in all groups was similar except that Group 1 received the (HR) base sentences alone, Group 2 received the HR-PE sentences, Group 3 received the HR-IE sentences, Group 4 received the LR-base sentences alone, Group 5 received the LR-PE sentences, and Group 6 received the LR-IE sentences. The subjects were distributed equally across the six conditions. After presentation of each of the 10 sentences orally, $5 \mathrm{sec}$ were allotted for a response in all groups.

After acquisition, all subjects were asked to count backward by threes for $60 \mathrm{sec}$. The subjects were then read the 10 base-sentence contexts (with a blank in place of the target word) as cues for the target words. After each cue was presented orally, $7 \mathrm{sec}$ were allotted for each response.

\section{Results}

An inspection of the incidental-task results indicated that the mean comprehension ratings for base, $\mathrm{PE}$, and IE sentences in the HR set were $3.64,3.92$, and 3.18, respectively. The mean comprehension ratings for base, $\mathrm{PE}$, and IE sentences in the LR set were 3.43, 3.51, and 4.06, respectively.

A summary of the mean number of items correctly recalled is presented in Table 1. A survey of the recall means indicates performance was highest for subjects receiving the PE sentences (Groups 2 and 5) followed by that for subjects receiving the base sentences (Groups 1 and 4) and subjects receiving the IE sentences (Groups 3 and 6).

A two-way analysis of variance on the recall scores using subjects $(F)$ and items $\left(F^{1}\right)$ as random effects revealed a significant effect for the type of elaborative context $\left[\mathrm{F}(2,78)=27.87, \mathrm{p}<.001, \mathrm{MS}_{\mathrm{e}}=3.73 ; \mathrm{F}^{1}(2,54)=\right.$ $\left.14.4, \mathrm{p}<.001, \mathrm{MS}_{\mathrm{e}}=9.25\right]$. The effect of sentence relatedness was not significant $[\mathrm{F}(1,78)=.156, \mathrm{p}>.65$; $\left.\mathrm{F}^{1}(1,54)=.001, \mathrm{p}>.90\right]$. The interaction between sentence relatedness and type of elaboration context was also not significant $\left[\mathrm{F}(2,78)=.711, \mathrm{p}>.45 ; \mathrm{F}^{1}(2,54)=\right.$ $.394, \mathrm{p}>.60]$. These findings indicate that the degree of target-elaboration relatedness had little effect on cuedrecall performance. Recall performance for subjects receiving the PE sentences (Groups 2 and 5) was significantly superior to that for subjects receiving the base sentences (Groups 1 and 4) [t(78) $=3.77, \mathrm{p}<.001]$, indicating that precise elaborations did enhance cued-recall performance. Recall performance for subjects receiving the IE sentences (Groups 3 and 6) was not significantly different from that of subjects receiving only the base sentences (Groups 1 and 4 ), [t(78) $=1.32$, p $>.05]$, indicating that imprecise semantically congruous elaborations did not facilitate cued-recall performance.

\section{Discussion}

The results of Experiment 1 demonstrate that elaborative information facilitated the retention of target concepts only when it helped clarify the precise significance of those words in the acquisition sentences. The associative relatedness of elaborative information and target concepts did not affect target retention. Adding imprecise elaborations that were either related or unrelated to the target

Table 1

Mean Number of Items Recalled: Experiment 1

\begin{tabular}{cccc}
\hline & Base & PE & IE \\
\hline HR & 4.14 & 7.29 & 3.79 \\
LR & 4.64 & 7.00 & 3.07 \\
\hline
\end{tabular}

Note-Base $=$ base sentences; $P E=$ precise sentences; $I E=$ imprecise sentences; $H R=$ high-related sentences; $L R=$ low-related sentences. 
concepts did not facilitate cued-recall performance. These findings differ from those of Fisher and Craik (1980), who found that the associative relatedness of elaborations to target concepts facilitated recognition. The present results suggest that associative relatedness is not sufficient to facilitate recollection in a cued-recall test.

It seems clear that there are a number of ways to define associative relatedness. In Experiment 1, elaborations were considered to be associatively related if they were highly related to target concepts. For example, in the sentence "The funny man bought the ring and told a joke," there is a strong relationship between "funny" and "joke." One could argue that these types of relationships do not provide sufficient opportunities for redintegration because they do not involve the base-sentence contexts (e.g., "buying a ring") and as a result cannot be easily redintegrated when the base-sentence context is given as a retrieval cue. To evaluate this hypothesis, Experiment 2 used elaborations that were related to both the target concepts and the base-sentence contexts. For example, the sentence "The rich man lifted the chair beside the expensive desk" included an elaborative concept "expensive desk" that was related to both the target "rich" and the base-sentence concept "chair."

\section{EXPERIMENT 2}

\section{Method}

Subjects. Eighty-four undergraduates enrolled in introductory psychology courses at Tennessee Technological University participated for course credit.

Materials. Two sets of sentence types were constructed. Each set contained base sentences (base), precise elaborations of these base sentences (PE), and imprecise elaborations of these base sentences (IE). In the first set, PE and IE sentences were constructed so that there would be a relatively high degree of associative relatedness among target concepts, base sentences, and words in the elaborative phrases. We refer to this as the high-related (HR) set. In the second set, PE and IE sentences were constructed so that there would be a lower degree of associative relatedness among target concepts, base sentences, and words in the elaborative phrases. We refer to this as the low-related (LR) set. Examples of base, PE, and IE sentences for the HR set are: "The rich man picked up the chair," "The rich man picked up the chair and looked at the gold legs," and "The rich man picked up the chair beside the expensive desk." Examples of base, PE, and IE sentences for the LR set are: "The short man bought the broom," "The short man bought the broom to operate the light switch," and " The short man bought the broom to sweep the floor." Both the HR and LR sentence sets are presented in Appendix 2.

Twenty subjects were given the target words paired separately with key concepts or phrases used in the PE and IE elaborations and concepts in the base-sentence contexts, for both the HR and LR sets, and were asked to rate the relatedness of each pairing on a scale of 1-5 (with 5 corresponding to high relatedness). The mean ratings for each sentence in PE and IE contexts of the HR set were 2.90 and 3.08, respectively. The mean ratings for the PE and IE contexts in the LR set were 1.91 and 2.38 , respectively. A $2 \times 2$ analysis of variance on the rating scores revealed a significant effect for relatedness (LR vs. HR) $[F(1,57)$ $\left.=162.21, \mathrm{p}<.001, \mathrm{MS}_{\text {Res }}=.087\right]$, indicating that $\mathrm{HR}$ sentences were generally rated higher than LR sentences. There were also sig nificant effects for the type of elaboration (PE vs. IE) and the interaction (PE-IE $\times$ HR-LR) $[\mathrm{F}(1,57)=24.29, \mathrm{p}<.001$, and $\mathrm{F}(1,57)=$ $4.83, p<.05$, respectively]. These latter findings are a result of the fact that IE sentences in the LR set were rated significantly higher than
PE sentences in the LR set $[F(1,57)=25.4, p<.001]$. There was no significant difference between the ratings for IE sentences and $\mathrm{PE}$ sentences in the HR set $[F(1,57)=3.72, p>.05]$.

An additional analysis was performed using the ratings assigned to the weakest relationship for each sentence. For example, in the sentence "The rich man lifted the chair beside the expensive desk," the relationship between "chair" and "expensive desk" might be weaker than that between "rich man" and "expensive desk." Subjects' ratings were used to select the weakest relationship in each sentence. The means for the IE and PE sentences in the HR set were 2.36 and 2.28, respectively. The means for $\mathrm{IE}$ and $\mathrm{PE}$ sentences in the LR set were 1.54 and 1.37 , respectively. A $2 \times 2$ analysis of variance on the low ratings for each sentence revealed a significant effect for relatedness (HR vs. $\operatorname{LR})\left[\mathrm{F}(1,57)=147.31, \mathrm{p}<.001, \mathrm{MS}_{\text {Res }}=.101\right]$, indicating that $H R$ sentences had higher relatedness ratings than $L R$ sentences when the weakest relationship in each sentence was considered. No other effects were significant

Procedure. The procedure was identical to that in Experiment 1.

\section{Results}

An inspection of the incidental-task results indicated that subjects in all groups rated the sentences similarly with respect to comprehensibility. The mean comprehension ratings for base, PE, and IE sentences in the HR set were $3.71,3.69$, and 3.46 , respectively. The mean comprehension ratings for base, PE, and IE sentences in the LR set were $3.94,3.28$, and 3.82 , respectively.

A summary of the mean number of items correctly recalled is presented in Table 2. A survey of the recall means indicates performance was highest for subjects receiving the PE sentences (Groups 2 and 5), followed by that for subjects receiving the base sentences (Groups 1 and 4) and subjects receiving the IE sentences (Groups 3 and 6).

A two-way analysis of variance on the recall scores using subjects $(F)$ and items $\left(F^{1}\right)$ as random effects revealed a significant effect for the type of elaborative context $\left[\mathrm{F}(2,78)=8.40, \mathrm{p}<.01, \mathrm{MS}_{\mathrm{e}}=4.38 ; \mathrm{F}(2,54)=4.61\right.$, $\left.\mathrm{p}<.05, \mathrm{MS}_{\mathrm{e}}=11.2\right]$. The effect of sentence relatedness was not significant $[F(1,78)=.044, p>.80$; $F(1,54)=.024, p>.80]$. The interaction between sentence relatedness and type of elaboration context was also not significant $\left[F(2,78)=.590, p>.50 ; F^{1}(2,54)=\right.$ $.324, \mathrm{p}>.70 \mathrm{]}$. These findings indicate that the degree of target-elaboration relatedness had little effect on cuedrecall performance. Recall performance for subjects receiving the PE sentences (Groups 2 and 5) was significantly superior to that for subjects receiving the base sentences (Groups 1 and 4 ) [t(78) $=2.68, p<.05]$, indicating that precise elaborations did enhance cued-recall performance. Recall performance for subjects receiving the IE sentences (Groups 3 and 6) was not significantly different from that of subjects receiving only the base sentences (Groups 1 and 4 ) $[t(78)=1.34, p>.05]$, indicat-

Table 2

Mean Number of Items Recalled: Experiment 2

\begin{tabular}{cccc}
\hline & Base & PE & IE \\
\hline HR & 4.64 & 5.86 & 4.21 \\
LR & 4.57 & 6.36 & 3.5 \\
\hline
\end{tabular}

Note-See Table I for definitions of abbreviations. 
ing that imprecise semantically congruous elaborations did not facilitate cued-recall performance.

\section{Discussion}

The results of Experiment 2 demonstrate that elaborations that clarify the significance of target concepts (PEs) facilitate cued-recall performance. The associative relatedness of elaborations to target concepts and basesentence concepts did not affect cued-recall performance. The latter result was found even though elaborations were related to both target concepts and base-sentence concepts. These findings suggest that the associative strength of preexisting relationships had no effect on retention in Experiment 2 . Instead, memory was influenced by the degree to which elaborative information clarified the significance of facts.

It is important to note, however, that in Experiments 1 and 2 the elaborations were not provided at the time of test. Recent investigations (e.g., Fisher, 1981; Fisher \& Craik, 1980) have shown that, depending on their availability at the time of testing, particular types of elaborations may or may not facilitate retention. It is possible, therefore, that imprecise elaborations that are associatively related to target concepts can facilitate retention when they are provided in the testing context. Experiment 3 investigated this issue by using a recognition procedure in which the entire acquisition sentence was presented during testing.

\section{EXPERIMENT 3}

\section{Method}

Subjects. Eighty-four undergraduates enrolled in introductory psychology courses at Tennessee Technological University participated for course credit.

Materials. The materials were the same as those in Experiment 2.

Procedure. The procedure utilized an acquisition phase identical to that of Experiment 2. Following acquisition, all subjects counted backward by threes for $90 \mathrm{sec}$. All groups were then given a recognition test. The test consisted of 30 orally presented sentences, 10 of which were sentences heard during acquisition. The 20 foils were constructed by re-pairing target concepts and acquisition-sentence contexts. The subjects were instructed to respond with a "yes" or a "no" as quickly as possible after each sentence had been presented to indicate whether they had heard it during acquisition. Separate recognition tests were used for the LR sets and the HR sets.

\section{Results}

An inspection of the incidental-task results indicated that subjects in all groups rated the sentences similarly with respect to comprehensibility. The mean comprehension ratings for base, $P E$, and IE sentences in the HR set were $4.19,4.22$, and 3.84 , respectively. The mean comprehension ratings for base, PE, and IE sentences in the LR set were $3.54,4.24$, and 3.74 , respectively.

A summary of the mean number of target sentences correctly recognized and the mean number of false positives for each condition is presented in Table 3.

A survey of the recognition hit rates indicates performance was highest for subjects receiving the PE sentences
Table 3

Mean Hit (H) Rates and False-Positive (FP) Rates

\begin{tabular}{|c|c|c|c|c|c|c|}
\hline & \multicolumn{2}{|c|}{ Base } & \multicolumn{2}{|c|}{ PE } & \multicolumn{2}{|c|}{ IE } \\
\hline & $\mathbf{H}$ & FP & $\mathrm{H}$ & $\overline{F P}$ & $\mathrm{H}$ & $\overline{\text { FP }}$ \\
\hline HR & 7.07 & 4.57 & 8.57 & .857 & 7.57 & 4.79 \\
\hline LR & 6.57 & 3.79 & 9.57 & .714 & 6.64 & 4.07 \\
\hline
\end{tabular}

(Groups 2 and 5), followed by that for subjects receiving IE sentences (Groups 3 and 4) and subjects receiving the base sentences (Groups 1 and 4).

A two-way analysis of variance was performed on the hit rates using subjects $(F)$ and items $\left(F^{1}\right)$ as random effects. A significant effect for type of elaboration (base vs. IE vs. PE) was found $[\mathrm{F}(2,78)=14.4, \mathrm{p}<.001$, $\left.\mathrm{MS}_{\mathrm{e}}=2.92 ; \mathrm{F}^{1}(2,54)=9.07, \mathrm{p}<.001, \mathrm{MS}_{\mathrm{e}}=4.57\right]$. The effect of sentence relatedness was not significant $\left[\mathrm{F}(1,78)=.147, \mathrm{p}>.70 ; \mathrm{F}^{1}(1,54)=1.05,, \mathrm{p}>.30\right]$. The interaction was also not significant $[\mathrm{F}(2,78)=2.46$, $\left.p>.09 ; F^{1}(2,54)=.900, p>.40\right]$. These findings indicate that the degree of sentence relatedness had little effect on hit rates. Recognition hit rates for subjects receiving PE elaborations (Groups 2 and 5) were significantly higher than those for subjects receiving base sentences (Groups 1 and 4) [t(78) $=3.48, p<.01]$, indicating that precise elaborations enhanced recognition hit rates. Recognition hit rates for subjects receiving IE elaborations (Groups 3 and 6) were not significantly different from those for subjects receiving base sentences (Groups 1 and 4$)[t(78)=.449, p>.05]$, indicating that imprecise elaborations that were either related or unrelated did not facilitate recognition hit rates.

An additional analysis of $D^{\prime}$ scores was performed to take false-positive scores into account. A data transformation involving the subtraction of 0.5 from all hit-rate scores and the addition of 0.5 to all false-positive scores was performed to reduce skewness. The mean $D^{\prime}$ scores for each group are presented in Table 4. A two-way analysis of variance of the $\mathrm{D}^{\prime}$ scores revealed a significant effect for type of elaboration (base vs. IE vs. PE) $[F(2,78)$ $\left.=26.9, \mathrm{p}<.001, \mathrm{MS}_{\mathrm{c}}=.579\right]$. The effect for relatedness was also significant $[F(1,78)=5.65, \mathrm{p}<.05]$. The interaction was not significant $[\mathrm{F}(2,78)=1.11, \mathrm{p}>$.30].

Recognition performance for subjects receiving the $\mathrm{PE}$ sentences in both the HR and LR conditions was significantly superior to that for subjects receiving the base sentences in the HR and LR conditions [ $t(78)=4.31, p<$ .01 , and $t(78)=4.28, p<.01$, respectively]. The latter result indicates that precise elaborations facilitated recognition performance in both the HR sentence sets and the LR sentence sets. Recognition performance for subjects

Table 4

\begin{tabular}{|c|c|c|c|}
\hline & Base & $\mathrm{PE}$ & IE \\
\hline HR & 1.26 & 2.50 & 1.41 \\
\hline LR & 1.83 & 3.06 & 1.46 \\
\hline
\end{tabular}

Note-See Table 1 for definitions of abbreviations. 
receiving the IE sentences in both the HR and LR conditions was not significantly different from that of subjects receiving only the base sentences in the HR and LR conditions $[\mathrm{t}(78)=.522, \mathrm{p}>.05$, and $\mathrm{t}(78)=1.29$, $\mathrm{p}>.05$, respectively], indicating imprecise elaborations did not facilitate recognition performance in either the HR or the LR condition.

The significant effect of relatedness on recognition performance was due in part to the subjects in the PE-LR condition performing better than the subjects in the PEHR condition. The subjects in the base-LR condition also seemed to perform better than the subjects in the baseHR condition. Neither of these comparisons was significant.

As suggested by one reviewer, the superior recognition performance shown by the subjects in the PE-HR and PE-LR conditions of Experiment 3 may be due to the fact that only the target sentences in the recognition test had a nonarbitrary relationship between the base sentences and their elaborations. Therefore, the subjects could have eliminated foil sentences on the basis of the arbitrariness of the relationship between the base sentence and the elaboration, rather than by recognizing the target sentence as having been heard before. To evaluate this possibility, two additional groups were tested. One received PEHR sentences at acquisition, and the other received PE-LR sentences. The acquisition sentences and procedure were identical to those used in the PE-HR and PE-LR conditions in Experiment 3. The recognition test was also identical, except that five of the foil sentences were replaced with sentences that were precisely elaborated (i.e., they had a nonarbitrary relationship between the target concept and the elaboration). For example, the foil sentence "The thin man picked up the chair and looked at the gold legs" was replaced by the precisely elaborated foil "The thin man purchased the weights to do some bodybuilding." If subjects performed better on the recognition test in the PE-HR and PE-LR conditions in Experiment 3 because they responded "yes" only to those sentences that had a nonarbitrary relationship between the target concept and the elaboration, the five PE foils should be falsely recognized more than the other foil sentences in the recognition test. In addition, the number of false positives should be higher than those for the corresponding conditions in Experiment 3.

The inclusion of PE foils in the recognition test had no effect on either the hit rates or the number of false positives identified in either the PE-HR or PE-LR groups. The mean number of hits for the PE-HR group was 9.33, and that for the PE-LR group was 9.18. The means for Experiment 3 were 8.57 and 9.57 for the PE-HR and PELR groups, respectively. The mean number of false positives identified was .83 for the PE-HR group and .82 for the PE-LR group. For Experiment 3, the mean for the false positives was .857 for the PE-HR group and was .714 for the PE-LR group. None of the five PE foil sentences in either condition were falsely identified as target sentences by any subject.

\section{DISCUSSION AND SUMMARY}

The results of Experiment 3 demonstrate that recognition performance was enhanced by elaborations only when the elaborations clarified the significance of target concepts (e.g., PEs). Elaborations that did not reduce the arbitrariness of relationships had little effect on retention. That is, semantically congruous elaborations that increased the complexity and distinctiveness of the memory trace had no effect on retention when the elaborations were not precise. These results were found under conditions in which the elaborations, target concepts, and base sentences were presented together at the time of test. These findings, together with those reported in Experiments 1 and 2, replicate and extend the findings of previous research (Stein et al. 1978; Stein \& Bransford, 1979) by showing that elaborations that modify the arbitrariness of relationships affect retention under varying degrees of associative relatedness.

The results of the three experiments reported in the present paper differ from those reported by Fisher (1981) and Fisher and Craik (1980). They found that elaborations that increased trace complexity and distinctiveness improved retention. However, these effects depended on the elaborations' being either (1) well-integrated with target concepts or (2) available at the time of test. Fisher and Craik used elaborations that were either associatively related to target concepts or unrelated to target concepts (see also Rosenberg, 1969). The present studies also varied associative relatedness of elaborations and target concepts. However, our studies show that elaborations that increase complexity do not necessarily increase retention even when they are available at the time of test, and that the associative relatedness of elaborations to to-beremembered information is neither necessary nor sufficient for retention.

One important reason for the differences in results reported by Fisher and Craik (1980) and the present study involves the types of materials used to investigate elaborative processes. The present study sought to investigate elaborative processes that might be needed to gain expertise and mastery in a new domain of knowledge. Therefore, materials were used that would provide an analog to the kinds of problems faced by novices trying to learn about a new domain. We have argued elsewhere (Bransford, Stein, Shelton, \& Owings, 1981; Stein \& Bransford, 1979) that novices frequently confront the problem of learning relationships that intially seem arbitrary. In order to investigate elaboration processes that are relevant to these situations, the present study used materials that were inherently more arbitrary than those employed by Fisher (1981) and Fisher and Craik (1980). Our results suggest that the arbitrariness of the materials to be learned constrains the types of elaborations that will be effective. Theories of elaboration must therefore consider the relationship between the materials to be learned and the knowledge that learners have available. For example, a learner who is quite familiar with a knowledge 
domain may produce effective elaborations if instructed to "generate elaborations that are associatively related to target concepts" (e.g., see Rosenberg, 1969). For a novice, however, existing associative relationships will not necessarily facilitate retention. Instead, novices must often be helped to use previously unrelated aspects of their knowledge in order to create elaborations that help them understand the significance or relevance of events (Bransford, Stein, Vye, Franks, Auble, Mezynski, \& Perfetto, 1982; Stein, Bransford, Franks, Owings, Vye, \& McGraw, 1982).

The present results also illustrate the need to clarify the concept of "distinctiveness." One could argue that elaborations that render relationships less arbitrary increase the distinctiveness of the memory trace. By the same token, imprecise elaborations could also be said to increase distinctiveness provided that the features activated are unique. If distinctiveness is assumed to increase as a function of the number of unique features that are activated, the present data reveal inadequacies in the theoretical construct. In particular, elaborations that increase distinctiveness do not always facilitate the retention of concepts involved in semantic relationships (see also Hunt \& Einstein, 1981). As an alternative, we emphasize the importance of elaborating in ways that establish unique relationships among concepts rather than in ways that merely increase the distinctive character of elements involved in those relationships. Elaborations that help people understand the significance of relationships therefore help them remember relational information as well as specific information about the key concepts involved in the relationship. The present results show that elaborations that enhance both relational and item-specific information do not require the activation of associatively related concepts that learners have previously acquired.

\section{REFERENCES}

ANDERson, J. R., \& REDER, L. M. (1979). An elaborative processing explanation of depth of processing. In L. S. Cermak, \& F. I. M. Craik (Eds.), Levels of processing and human memory. Hillsdale, NJ: Erlbaum.

AUble, P. M. (1982). An examination of expertise. Unpublished manuscript, Vanderbilt University, Nashville, TN.

Bransford, J. D., Stein, B. S., Shelton, T. S., \& Owings, R. A. (1981). Cognition and adaptation: The importance of learning to learn. In J. Harvey (Ed.), Cognition, social behavior and the environment. Hillsdale, NJ: Erlbaum.

Bransford, J. D., Stein, B. S., Vye, N. J., Franks, J. J., Auble, P. M., Mezynski, K. J., \& Perfetto, G. A. (1982). Differences in approaches to learning: An overview. Journal of Experimental Psychology: General, 111, 390-398.

Chiesi, H. L., Spilich, G. J., \& Voss, J. F. (1979). Acquisition of domain-related information in relation to high and low domain knowledge. Journal of Verbal Learning and Verbal Behavior, 18, 257-273.

Craik, F. I. M., \& Tulving, E. (1975). Depth of processing and the retention of words in episodic memory. Joumal of Experimental Psychology: General, 104, 269-294.

FISHER, R. P. (1981). Interaction between encoding distinctiveness and test conditions. Journal of Experimental Psychology: Human Learning and Memory, 7, 306-310.
Fisher, R. P., \& CRAIK, F. I. M. (1980). The effects of elaboration on recognition memory. Memory \& Cognition, 8, 400-404.

HunT, R. R., \& EInstein, G. O. (1981). Relational and item-specific information in memory. Journal of Verbal Learning and Verbal Behavior, 20, 497-514.

Johnson-LAIRD, P. M., \& Bethell-Fox, C. E. (1978). Memory for questions amount of processing. Memory \& Cognition, 6, 496-501.

RoHWER, W. D., JR. (1966). Constraints, syntax and meaning in pairedassociate learning. Journal of Verbal Learning and Verbal Behavior, 5, 541-547.

ROHWER, W. D., JR. (1980). An elaborative conception of learner differences. In R. E. Snow, P. A. Frederico, \& W. E. Montague (Eds.), Aptitude, learning, and instruction. Hillsdale, NJ: Erlbaum.

RoSENBERG, S. (1969). The recall of verbal material accompanying semantically well-integrated sentences. Joumal of Verbal Learning and Verbal Behavior, 8, 732-736.

Ross, B. H. (1981). The more, the better?: Number of decisions as a determinant of memorability. Memory \& Cognition, 9, 23-33.

SteIN, B. S., \& BranSFORD, J. D. (1979). Constraints on effective elaboration: Effects of precision and subject generation. Journal of Verbal Learning and Verbal Behavior, 18, 769-777.

Stein, B. S., Bransford, J. D., Franks, J. J., Owings, R. A., Vye, N. J., \& McGraW, W. (1982). Differences in the precision of selfgenerated elaborations. Journal of Experimental Psychology: General, 111, 399-405.

Stein, B. S., Morris, C. D., \& Bransford, J. D. (1978). Constraints on effective elaboration. Journal of Verbal Learning and Verbal Behavior, 17, 704-714.

Appendix 1

Acquisition sentences for Experiment 1 (Words and Phrases Used to Obtain Ratings of Associative Relatedness Are Italicized)

Base Sentences and Precise HR (in Parentheses)

1. The short man put up the tent (that was 2 feet high).

2. The funny man bought the ring (that squirted water).

3. The strong man read the book (about weight lifting).

4. The grey-haired man carried the bottle (of hair dye).

5. The fat man went to the train station (and got stuck in the gate).

6. The rich man bought the paint (to change the color of his yacht).

7. The hungry man bought a tie (to get into the fancy restaurant).

8. The tall man purchased the crackers (that were on the top shelf).

9. The smart man went to work (before rush hour).

10. The bald man used the telephone (to find out about the hat sale).

Base Sentences and Imprecise-HR (in parentheses)

1. The short man put up the tent (and walked under the low sign).

2. The funny man bought the ring (and told a joke).

3. The strong man read the book (and lifted weights).

4. The grey-haired man carried the bottle (and had his hair dyed).

5. The fat man went to the train station (and got something to eat).

6. The rich man bought paint (and went out on his yacht).

7. The hungry man bought a tie (and went to the grocery store).

8. The tall man purchased the crackers (and looked on the top shelf).

9. The smart man went to work (and planned his next investment).

10. The bald man used the telephone (and looked for a hat).

Base Sentences and Precise-LR (in parentheses)

1. The short man picked up the broom (to operate the light switch).

2. The funny man bought a bathtub (to use as a punch bowl).

3. The kind man ate dinner (and offered to wash the dishes).

4. The thin man found the scissors (and cut the belt in half).

5. The fat man wore the shoes (out in one week).

6. The rich man picked up the chair (to burn in his fireplace).

7. The sad man admired his new boat (and tried to fix the hole in the side).

8. The tall man got a hair cut (and helped the barber off the stool).

9. The smart man used the charcoal (when the pen broke).

10. The lucky man walked home (before it rained). 
Base Sentences and Imprecise-LR (in parentheses)

1. The short man picked up the broom (to sweep the floor).

2. The funny man bought a bathtub (to put in his bathroom).

3. The kind man ate dinner (in the evening).

4. The thin man found the scissors (and cut the paper).

5. The fat man wore the shoes (out in the rain).

6. The rich man picked up the chair (to move it).

7. The sad man admired his new boat (and looked at the crew).

8. The tall man got a hair cut (and helped the barber clean up).

9. The smart man used the charcoal (to cook dinner).

10. The lucky man walked home (before going to bed).

Appendix 2
Acquisition Sentences for Experiments 2 and 3 (Words
and Phrases Used to Obtain Ratings of Associative
Relatedness are Italicized)
Base Sentences and Precise-HR (in parentheses)
1. The short man bought the broom (to sweep the crawl space).
2. The brave man gave money to the robber (before he shot him).
3. The fat man read the sign (warning about thin ice).
4. The tall man bought the crackers (that were on the top shelf).
5. The thin man found the scissors (and cut the skinny belt in half).
6. The rich man picked up the chair (and looked at the gold legs).
7. The sad man looked at his new boat (that had been destroyed by
a tidal wave).
8. The kind man ate dinner (and offered to help wash the dishes).
9. The smart man went to work (and made a wise investment).
10. The bald man used the phone (to call about the hat sale).
Base Sentences and Imprecise-HR (in parentheses)
1. The short man bought the broom (to sweep a small pile of dirt).
2. The brave man gave money (to save the sick person).
3. The fat man read the sign (with the big letters).
4. The tall man bought the crackers (and then looked at the top shelf).

5. The thin man found the scissors (and cut the skinny thread).

6. The rich man picked up the chair (beside the expensive desk).

7. The sad man looked at his new boat (with the unhappy fisherman).

8. The kind man ate dinner (and had a generous serving).

9. The smart man went to work (in the productive factory).

10. The bald man used the phone (with the shiny surface).

Base Sentences and Precise-LR (in parentheses)

1. The short man bought the broom (to operate the light switch).

2. The funny man bought a bathrub (to use as a punch bowl).

3. The strong man talked to the woman (about moving her air conditioner).

4. The lucky man walked home (before it rained).

5. The thin man found the scissors (and put another hole in his belt).

6. The rich man picked up the chair (to burn in the fireplace).

7. The sad man looked at his new boat (and tried to fix the hole in the side).

8. The frightened man walked up the steps (to avoid the elevator).

9. The smart man used the charcoal (when the pen broke).

10. The brave man went into the candy store (that was on fire).

Base Sentences and Imprecise-LR (in parentheses)

1. The short man bought the broom (to sweep the floor).

2. The funny man bought a bathtub (on his way home from work).

3. The strong man talked to the woman (during dinner).

4. The lucky man walked home (and watched the news).

5. The thin man found the scissors (and put another hole in the paper).

6. The rich man picked up the chair (to sit in front of the fireplace).

7. The sad man looked at his new boat (and went shopping).

8. The frightened man walked up the steps (because the elevator wasn't working).

9. The smart man used the charcoal (and drank the water).

10. The brave man went into the candy store (and paid the clerk). (Manuscript received March 28, 1984; revision accepted for publication June 20, 1984.)

\section{ANNOUNCEMENT}

\section{Change in Editorship of Memory \& Cognition}

Robert Bjork completes his regular 4-year term as editor of Memory \& Cognition in 1985. The Publications Committee of the Governing Board of The Psychonomic Society is pleased to announce that Alice F. Healy has agreed to be the next editor (1986-1989). After January 1, 1985, please send manuscripts to Dr. Healy at: Department of Psychology, University of Colorado, Muenzinger Building, Campus Box 345, Boulder, CO 80309. 